Mots. Les langages du politique

$81 \mid 2006$

Suisse, laboratoire politique européen ?

\title{
Couleurs, fleurs et drapeaux dans les débuts de la Troisième République
}

\section{Maurice Tournier}

\section{(2) OpenEdition \\ Journals}

Édition électronique

URL : https://journals.openedition.org/mots/17093

DOI : $10.4000 /$ mots. 17093

ISSN : 1960-6001

Éditeur

ENS Éditions

Édition imprimée

Date de publication : 1 juillet 2006

Pagination : 109-117

ISBN : 2-84788-098-4

ISSN : 0243-6450

Référence électronique

Maurice Tournier, "Couleurs, fleurs et drapeaux dans les débuts de la Troisième République », Mots. Les langages du politique [En ligne], 81 | 2006, mis en ligne le 01 juillet 2008, consulté le 23 avril 2022. URL: http://journals.openedition.org/mots/17093 ; DOI : https://doi.org/10.4000/mots.17093 


\section{MOTS ET SYMBOLES EN POLITIQUE}

\section{Couleurs, fleurs et drapeaux dans les débuts de la Troisième République}

Nombreux sont en politique les désignants et même les collectifs qui glorifient ou stigmatisent et qui, tout en jouant par ailleurs et souvent en même temps le rôle de label, contribuent au marquage affectif ou moral des désignés. Avec les désignants polémiques propres au scandale du Panama, avec les appellations antidreyfusardes et antisémites, nous avons atteint une couche plus pure de ce type de vocabulaire. Nous avons aperçu aussi l'usage que l'on a pu faire des couleurs du drapeau national. Reprenons plus en détail cette question, en commençant par les évaluations que certaines couleurs ont pu propager sous la Troisième République ${ }^{1}$. Tout affrontement a ses symboles, ses étendards et ses gerbes.

À cette époque, le blanc aurait dû être l'apanage des seuls légitimistes si le dernier d'entre eux, comte de Chambord, dit Henri V, ne s'était pas absenté de la scène politique en refusant, à la différence des orléanistes tricolores, tout emblème républicain. En conséquence, la couleur blanche a vu son usage s'élargir à l'ensemble du monarchisme jusqu'à en devenir le linceul. Le Drapeau du 15 janvier 1899 en retrace les origines :

Charles IX et Henri III portant rouge, les protestants prirent le blanc olivin de la liberté de conscience, origine du panache blanc d'Henri IV, de sorte que le blanc [...] devint la couleur du royaume de France.

Son usage s'est même étendu bien au-delà : on parlera de " syndicats blancs » (Casals, 1978, p. 59), de " comités blancs », de «blanques orelles » ou «blandezingues » (rouffions antigrévistes et faux patriotes) ${ }^{2}$, d' « Fillet blanc » ${ }^{3}$, voire de «Café blanc » opposé au « Café rouge » (Daudet, 1898, p. 325-326), etc.

1. Mettons évidemment à part l'usage raciste des couleurs (sang rouge, sang noir, "variété mélanienne », "sang civilisateur ", "sang arian », race blanche, peuples noirs, les Jaunes, les Noirs, les Blancs, etc., chez Gobineau par exemple). Voir Pierre-André Taguieff, 1998, chap. 2, p. 21-58.

2. Selon le lieutenant $Z^{*}$ dans L'Armée aux grèves (1904), cité par Émilien Carassus, 1982, p. 44, note 45 .

3. «C'est la débandade, et le bel élan de l’CEillet blanc est arrêté. » (Le Radical, 12 juin 1899.)

Directeur de recherches émérite au CNRS, maurice.tournier@wanadoo.fr 
L'œillet, blanc comme lys, symbole de la droite nationaliste antirépublicaine $^{4}$, est le signe de ralliement des «bandes de l'Fillet blanc, des nationalistes et des antisémites », selon Le Radical du 12 juin 1899, qui constate après la bagarre d'Auteuil ${ }^{5}$ :

Ces messieurs de l'๔illet blanc et de l'Æillet rouge ${ }^{6}$, fauteurs de désordre [...], soldats de Déroulède ou amis de Guérin ou affiliés des comités bonapartistes ou membres de la jeunesse royaliste, troublent la rue.

Cela au nom du candidat unifié des monarchistes, Henri d'Orléans, surnommé «Gamelle » chez les conscrits. Celui-ci, tout en restant bleu (bleu que les nationalistes reprennent à leur compte, car «c'est lui qui tient le drapeau français à la hampe »), se veut tricolore, à l'instar de son ancêtre Louis-Philippe, mais devient blanc pour ses adeptes. Le Radical en vient à plaindre la fleur: «Pauvre œillet, toujours réactionnaire! Rouge avec Boulanger, bleu avec Drumont, blanc avec Gamelle. »7

C'est dire aussi l'ambigüité et les mutations du bleu (Pastoureau, 2000), couleur républicaine ${ }^{8}$ après avoir été celle de Charlemagne et des Capétiens (d'où le « sang bleu » et le ruban bleu des rois de France) ${ }^{9}$, mais devenue aussi récemment, avec l'affaire Dreyfus, couleur de l'œillet piqué à la boutonnière des activistes d'extrême droite : "L'œillet bleu est la fleur antisémite », commente Le Radical. On dit aussi "les saphirs» pour les antisémites de «la Haute»: « Fillets blancs et saphirs, telle aura été la devise des derniers conspirateurs de ce siècle. ${ }^{10}$ En général pourtant, blanc et bleu s’opposent encore, comme ils s’opposaient pendant la guerre de Vendée. D’où la réaffirmation colorée de son antimonarchisme par Paul de Cassagnac, bonapartiste grand teint :

4. La «Société de l'đFillet blanc» est une association monarchiste : "À ce complot auraient pris part la Société de l’CEillet blanc et les royalistes... », déclare Cassagnac au Parlement (selon L'Autorité, 7 juin 1899).

5. Il s'agit de l'agression qu'Émile Loubet a subie de la part du baron Cristiani. Charles Dupuy en fait le récit au Parlement en ces termes : "C'est à ce moment qu'un membre de l'Fillet blanc s'est précipité et a porté un coup de canne au président de la République. » (cité par L'Autorité, 7 juin 1899.)

6. «La Ligue des Patriotes s'est rendue à Croissy pour y commémorer l'arrestation illégale et arbitraire de Paul Déroulède. [...] Les patriotes en nombre considérable, portant l'œillet rouge à la boutonnière, sont réunis. » (Le Drapeau,16 aout 1902.) Rien de commun avec l'œillet rouge communard chanté par Louise Michel...

7. 6 juin 1899. La fleur semble ici compter plus que la couleur. Face à une manifestation républicaine, « on cherche les œillets blancs, bleus ou rouges, ils sont en bien petit nombre » (Le Radical, 13 juin 1899).

8. «"Les bleus! Les bleus!” C'était le cri des Chouans quand ils apercevaient les citoyens de la République ou les soldats de Louis-Philippe. Malheur au bleu qui s'écartait un instant du gros des bataillons. » (Duckett éd., 1865, t. 3, p. 293)

9. «C'est de Pannonie que revinrent les Francs portant le manteau bleu de Saint-Martin. Ce fut l'origine du manteau bleu de Charlemagne, couleur glorieuse qui devint le ruban bleu des rois et des grands Capétiens. » (Le Drapeau, 15 janvier 1899)

10. L'lllustration, 22 juillet 1899. 
Je ne suis pas royaliste, je suis ce que j'ai été et ce que je serai toujours, c'est-à-dire impérialiste. [...] Bleu et petit-fils de bleu, je trouve malsain d'essayer d'établir des classes entre les divers patriotismes ${ }^{11}$.

À écouter Cassagnac, on voit que le bleu n'est pas révolutionnaire! Flaubert opposait, dans L'Éducation sentimentale (1869), « les rouges et les bleus, les furibonds et les tranquilles $"$.

On identifie mieux le rouge que le bleu, de loin comme de près, chez les ennemis comme chez les amis (Dubois, 1962, p.121-122 et 411-412). Romieu, auteur du Spectre rouge, démontrait en 1852 la nécessité d'un pouvoir fort capable de juguler la menace socialiste (Napoléon III le nommera directeur des Beaux-Arts $)^{12}$. De l'autre côté, quel symbole plus célébré, depuis Blanqui en février 1848, que le drapeau rouge! N'est-il pas « rouge du sang de l'ouvrier », dans la chanson célèbre créée par Paul Brousse pendant son exil genevois après la Commune? Écoutons un journal lyonnais, Le Peuple: "Ce soir flotte et flottera le drapeau rouge intact de ces souillures et de ces hontes [...], le drapeau de la révolution.» (10 septembre 1899) Quelle "révolution», au fait? Celle des "socialistes internationalistes, les "rouges" qui parcourent les rues de la cité ${ }^{13}$ ou celle, nationaliste, de Drumont-Torquemada et de ses lieutenants préfascistes ${ }^{14}$ ? À moins qu'il ne se produise des retournements de veste, reproche fait aux socialistes nationalistes de l'époque. Dédié à Camille Pelletan, voici le coquelicot, la «fleur des barricades » :

Honte à qui, changeant de toquade,
Passe de l'écarlate au blanc!
Quoique modérément sanglant,
Je suis la fleur de barricade. ${ }^{15}$

Les révolutionnaires sont de loin les mieux marqués. Bien d'autres fleurs que les « œillets rouges » et les coquelicots vermillonnent sur les poitrines lors des manifestations: tulipes et roses rouges ${ }^{16}$, églantines républicaines ${ }^{17}$, même

11. Discours au Parlement, 5 juin 1899.

12. Très présent encore à la fin du siècle, ce «spectre rouge, épouvantail des bourgeois » (Le Parti ouvrier, 14 janvier 1899).

13. Le Drapeau, 14 février 1903.

14. "Drumont est là dans son milieu. Ce nouveau Torquemada, ainsi que ses lieutenants rouges Régis et Guérin, sont bien les dignes comparses des officiers et des évêques. » (Le Parti ouvrier, 14 mai 1898)

15. Eloy Vincent, La Serre parlementaire [1912], dans Tillier, 1997, p. 135.

16. " [Les républicains] auront pour signe de ralliement une rose rouge à la boutonnière ou des fleurettes rouges. » (Le Radical, 12 juin 1899)

17. L'églantine n'est pas révolutionnaire mais républicaine : «Tous, hommes, femmes, enfants, ont arboré à la boutonnière ou au corsage la petite fleur rouge, signe de ralliement des républicains, c'est la rose des bois, l'églantine modeste. » (Le Radical, 13 juin 1899.) Légion d'honneur pour les instituteurs et surtout leurs inspecteurs : «La République n’a que son ruban de soie / Qui, comme une églantine entrouverte, flamboie / Sur la poitrine des vainqueurs. » Adolphe Vincent, «A. M. Platrier» (1904), cité dans Crozet, 1991, p. 386. Victor Hugo a été jugé digne de l'églantine par les communards (voir Tillier, La Républicature, p. 133). 
dévaluées par l'ambition des églantinards. Avec ce terme, Barrès désignera les socialistes. Dans La Libre Parole du 6 juin 1904, l'expression «églantinards de ministère » désigne, elle, les gouvernementalistes modérés gourmands de décorations, tels les chasseurs de légion d'honneur et de rosettes qui entourent Combes :

\author{
Églantinards de ministères, \\ Anarchistes de profession... \\ Voilà ton armée à tout faire, \\ Ton ton tontaine et ton ton. \\ (Augustin Louis, «Le chasseur de nonnes ») ${ }^{18}$
}

Bémol encore ! Car il existe aussi des roses roses, des socialistes rosés ${ }^{19}$ et des « républicains roses» (modérés), qui ne font pas forcément bon ménage avec les rouges : «[Les conservateurs] renonceront à mener une existence devenue intolérable ; ils iront attendre sous des cieux plus cléments la fin de la crise, et ils laisseront en présence les républicains roses et les républicains rouges. ${ }^{20}$ Mais tout dépend du point d'observation. Qui dit «Les rouges » peut désigner à l'époque tous les socialistes, même s'il s'agit d'un Millerand gouvernementable, sous la plume de Cassagnac par exemple: «Si les loups rouges menacent effectivement la bergerie, nous estimons que le chien de berger [Méline ?] est bien édenté. ${ }^{21}$ D'où l'indéfinition de la couleur pour certains. Paul Lafargue se gaussait des «indépendants polychromes du socialisme sentimental ». Est-ce pour cela que Le Figaro du 16 janvier 1895 dit de Jaurès, à l'Assemblée, que « planté au centre gauche [il] a fleuri socialiste ${ }^{22}$ ?

Ce cas est pourtant rare. Rouge signifie davantage révolutionnaire que réformiste. La « Messe rouge » est un autre nom du Grand Soir ; « Rouge était le soleil levant » annonce, chez Louise Michel, la venue du Grand Jour ${ }^{23}$. "Salut à la race au sang chaud et vermeil, en qui tout sera justice, harmonie, force et lumière! Vive la Commune morte!", écrira-t-elle dans ses Mémoires. Pas un $1^{\text {er }}$ Mai ouvrier depuis 1890 sans drapeaux, bengales et lampions rouges ni églantines, coquelicots, lilas, roses ou tulipes de même couleur (Rodriguez, 1990, p. 15, $152-158)^{24}$. Le rouge est devenu, dans le monde ouvrier hors frontières, la couleur de l'espérance: "Ils ont des drapeaux rouges parce que c'est le signe de ralliement universel des travailleurs qui espèrent » (Le Réveil $d u$ Nord, $1^{\text {er }}$ mai

18. Cité dans Barbier et Vernillat, 1961, t. 8, p. 179.

19. Nuances : «Dernièrement, le citoyen Besombes appelait, avec raison, les groupes de jeunesse des Toucouleurs. En effet, ils sont composés de tous les éléments, depuis le rouge le plus pâle jusqu'au rouge le plus vif. » (Le Parti ouvrier, 22 avril 1898.)

20. L'Autorité, 14 juin 1899.

21. L'Autorité, 15 juin 1898.

22. Cité dans Willard, 1967, p. 73.

23. Voir Tournier, 2002, vol. 1, p. 211-236.

24. Le muguet n'arrive qu'en 1913, d'ailleurs cravaté de rouge, et ne s'imposera qu'en 1940, tout blanc. 
1906) ; « Notre drapeau, c'est le drapeau international. C'est le drapeau rouge. Il ne porte pas d'inscription. Il a juste, dans un coin, un soleil levant. » (1899; ibid., p. 165.)

Tout proche de ce rouge sang se trouve le noir anarchiste, fomentateur de révolution, mais dans l'absolu opposé existe aussi le noir clérical et conformiste, bénisseur de répression. Le premier, ancien signal de la peste et du choléra, déjà brandi par les canuts lyonnais descendant de la Croix-Rousse en 1831 et en 1834, est devenu signe d'anarchie militante, se mêlant au drapeau rouge révolutionnaire dans les manifestations: "Une tiaulée de fistons délurés, décrit Le Père Peinard, ont tenu à affirmer leur haine du militarisme : encocardés de noir et de rouge, ils ne clamaient pas “Vive la classe!" mais simplement "Vive la liberté !" " (19 février 1899.) Dans les grèves ouvrières, le noir et le rouge se mêlent. Et les sergents de ville, selon Jules Jouy, de chanter :

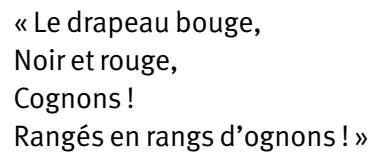

Entre les deux noirs, anarchiste et clérical, aux deux extrêmes du spectre sociopolitique, un usage majoritaire a tranché : au carrefour anticlérical de la presse révolutionnaire, anarchiste, socialiste et radicale (mais totalement absent de la littérature monarchiste), le noir, c'est d'abord et surtout la couleur honnie des autres, tout spécialement des «curés». Dans cette presse, cléricalisme et noir forment presque pléonasme : «Si nous ne nous réveillons pas, nous nous trouverons un beau matin liés sous l'abominable réseau noir des cléricailleries $»^{25}$; «Les taches noires [des congrégations] se plaquent sur le pays telle une lèpre envahissante ${ }^{26}$; pour Millerand, le cléricalisme " s'étend chaque jour davantage comme une tache noire sur le pays de France ${ }^{27}$; les «culs noirs " sont les Jésuites ${ }^{28}$; la « secte noire », le parti catholique ${ }^{29}$; la «bande noire » désigne tantôt le clergé ( abrutisseur des peuples $»^{30}$ ), tantôt les hommes d'affaires et la « réaction », " qui veut continuer son œuvre d'abêtissement et d'exploitation des masses ${ }^{31}$; au Grand Orient, reprenant la chanson de Pierre Jean de Béranger, l'« homme noir » est le prêtre ${ }^{32}$; à un congrès de la Bourse du

25. Jules Lermina, Le Radical, 18 novembre 1896.

26. Zo D’Axa, La Feuille, 3 novembre 1898.

27. Cité dans Le Parti ouvrier, 3 décembre 1898.

28. "S'ils sont de franches crapules, ils ne sont pas des truffes [imbéciles], les culs-noirs. » (Le Père Peinard, 3 juillet 1898.)

29. «De nombreux chefs de corps, affiliés à la secte noire, préparent depuis longtemps un coup d’État contre la République » (Allemane, Le Parti ouvrier, 5 mars 1898).

30. Le Parti ouvrier, 26 février 1898.

31. Le Parti ouvrier, 7 janvier 1899.

32. "C'est par la femme que notre ennemi le prêtre, l'homme noir, parvient à dominer la société » (25 janvier 1877). Cité dans Wartelle, 1993, p. 82. 
Travail de Paris, le SECI, syndicat catholique, est accusé d'être un syndicat noir et exclu. La Marseillaise anticléricale (1881) de Léo Taxil (G. A. Jogand-Pagès) a pour refrain :

\author{
Allons, fils de la République, \\ Le jour de vote est arrivé ! \\ Contre nous, de la noire clique \\ L'oriflamme ignoble est levé. ${ }^{33}$
}

Aucune espèce d'ambigüité, à l'évidence, entre le noir anarchiste et ce noir «obscurantiste» par définition: autant le premier est revendiqué, symbole du libertarisme, autant le second est repoussé ou ignoré par ses suppôts. Le premier est un drapeau dont on se fait gloire, le second se recouvre du drapeau blanc ou tricolore. Ce noir-là sert donc exclusivement à la dénonciation. Innombrables, les injures du type : pieuvre noire, cochons noirs, vermine noire, racaille noire, corbeaux, sacs à charbon, etc. On ne s'étonnera pas de constater l'antagonisme violent qui oppose à l'époque le rouge révolutionnaire au noir "clérico-réactionnaire»: "Il faut revenir à la franche distinction de Clemenceau et du comte de Mun, Révolution ou Église. Il faut être de l'Internationale rouge ou de l'Internationale noire. " ${ }^{34}$ Rarissime donc, l'oxymore curé rouge, appellation qui ne date que de 1901 (et non de la Révolution française, comme on l'a cru) (Geffroy, 1989, p 102-106) ${ }^{35}$. Moins rare, le label Jésuite rouge désigne les francs-maçons ou nomme Guesde.

Très curieusement, le noir clérical et le blanc monarchiste côtoient le jaune, qui devient, en cette fin du $19^{\mathrm{e}}$ siècle, la couleur puis le label des briseurs de grève et des syndicats "indépendants » et/ou patronaux, ligués au nom de la liberté du travail. Nous avons montré ailleurs ${ }^{36}$ que l'usage excluant de ce motcouleur ne date pas des grèves de Montceau-les-Mines et du Creusot (18991900) mais qu'il s'est enté, dès les années 1870, dans le discours ouvrier occidental, sur des mythes préexistants (antihérétiques) ou modernes (racistes et nationalistes). Insulte, jouant un rôle de marqueur en Occident du rejet social ${ }^{37}$ dès l'origine, le jaune s'est efforcé, par «rétorsion », de jouer celui d'étiquette officielle d'un syndicalisme de type antirévolutionnaire ${ }^{38}$, aidé, voire lancé, par le grand patronat. La Fédération nationale des Jaunes de France de Pierre Biétry date de mars 1902 ; Bamboigne (surnom d’Eugène Schneider fils) avait aupa-

33. Cité dans Barbier et Vernillat, 1961, p. 59.

34. Revue socialiste, mars 1892, p. 352.

35. Apparait dans Germinal le personnage du prêtre «rouge» (Ranvier) qui, disent les bourgeois, «trouvait des excuses aux scélératesses des grévistes».

36. La question a été traitée dans Tournier, 2002, vol. 1, p.181-205.

37. Cocarde des citoyens indélicats et ruban des «bambins indociles » sous la Révolution, pavillon de la quarantaine, couleur du cocu comme du forçat («J'ai le passeport jaune», dit le Jean Valjean des Misérables dès son arrivée à la cure de Montfermeil).

38. Voir sur ce point Sternhell, 1978, p. 245-317. 
ravant suscité au Creusot (en 1899) un "syndicat jaune » pour faire pièce à un « syndicat rouge » (Parize, 1999, p. 97-108).

Militants et adhérents peuvent se fleurir. Ils arborent alors au veston la fleur jaune du genêt. Le journal de P. Biétry, Le Jaune, ainsi que les Cahiers de l'Ouvrier, la font figurer en 1904 en haut de la première page à titre d'emblème. «Nos organisations se parent orgueilleusement de l'épithète décochée en pleine bataille. Notre insigne est le genêt ; celui des Rouges, l'églantine », écrit P. Biétry (1906, p. 83).

On ne compte plus, au tournant des siècles, les affrontements violents entre rouges, syndicalistes révolutionnaires, et jaunes, renards ou renégats (milices patronales, syndicats indépendants, nervis et hommes de main nationalistes) ${ }^{39}$, les « réformistes » étant hors champ ${ }^{40}$.

D’autres couleurs ont également habillé, dans le discours ouvrier, les ennemis des bourses et de la CGT; mais le jaune semble les avoir submergées avant de disparaitre, en tant que label fédéral, dès décembre 1902, certes au profit d' « indépendants » mais non en tant qu'injure sociale ${ }^{41}$, directe ou retourné $e^{42}$. Enfin, le vert des anciens verdets du duc d'Angoulême, en souvenir de 1815, a pu accompagner le jaune et le noir dans certaines manifestations nationalistes de la Ligue des Patriotes. Le Drapeau raconte : "Jacques Robert, délégué général de la LDP, assistait aux obsèques, accompagnant la couronne en immortelles jaunes avec rubans tricolores et aux couleurs verte et noire de la Ligue. ${ }^{43}$

Reste le tricolore ${ }^{44}$, signe républicain réofficialisé lors de l'affrontement public entre Alphonse de Lamartine et Auguste Blanqui en février 1848, mais aussi triade patriotique de couleurs, donc signe multiplié du ralliement français.

39. Autres appellations des anti-grévistes: bédouins, caffards, cosaques, fainéants, faux-frères, galvaudeux, italiens, kroumirs, macchabées, magots, pavillons-noirs, rats, rossards, salauds, sarrasins, traitres, mélange d'argots et de colonismes xénophobes. Voir Perrot, 1974, t. 2, p. 518519.

40. " On ne les appelle pas traîtres ni jaunes, car ils ne poussent pas le cynisme jusqu'à nier la lutte des classes. [...] Mais on les marque de l'épithète dédaigneuse de réformistes. » (Mermeix, 1907, p. 168.)

41. En 1906, par exemple : « Le patron, sûr de l'impunité, tue lui-même ses ouvriers et les fait assassiner par ses jaunes. » (Paul Lafargue, cité dans Brossat et Potel, 1975, t. 1, p. 117)

42. On peut constater à ce propos une curieuse ambivalence de l'étiquette: alors que certains jaunes enrégimentés et «bons Français », défenseurs du «travail national », comptent parmi les adversaires virulents des importations d' "étrangers» sur le marché de l'emploi, les Jaunes dénoncés comme briseurs de grève sont assimilés à ces étrangers. "Le nègre, le coolie chinois se profilent à l'horizon mental de la classe ouvrière. » (Perrot, 1974, p. 177-179).

43. 23 mai 1903. Symbolismes récurrents : Le Drapeau décrit aussi, au 14 juillet de la même année, "l'immense couronne de la LDP, en immortelles jaunes, cravatée de vert et de noir et d'un ruban tricolore voilé de crêpe». "Elle porte, ajoute-t-il, cette inscription: “À l’Alsace-Lorraine, la LDP”. » (18 juillet 1903.)

44. Le Drapeau du 15 janvier 1899 se plait à rappeler l'utilisation armoriale des trois couleurs au

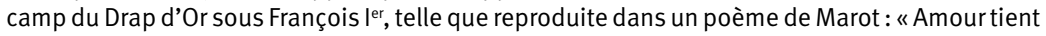
l'ung de couleur blanche et monde [Blanc pur] ; / Triomphe l'aultre avecque ses souldars / Vivement painst de couleur célestine [Bleu ciel]; / Beauté après en sa main noble et digne / Porte le tiers tainct de vermeille sorte [Rouge sang]. » 
Le drapeau national est tellement disputé qu'il permet de fleurir presque toutes les tendances, à l'exception des anarchistes et socialistes révolutionnaires. Les Jaunes agitent des drapeaux tricolores ${ }^{45}$. On dénonce à l'extrême gauche la «tricoloromanie»: «Tricoloromanie. Nous voici en pleine période quatorze-juilletarde, le moment où la rage tricolore sévit le plus » (Le Parti ouvrier, 16 juillet 1898). Et cela va jusqu'à la haine du drapeau ${ }^{46}$. À droite, les patriotes dénoncent ceux qui, par dreyfusisme, viennent « baver sur le drapeau » (L'Autorité, 26 novembre 1898). À cette époque, le mot tricolore signifie et authentifie " nationaliste ». C'est la couleur « hypocrite » avec laquelle les ouvriers en grève peignent leurs patrons républicains : «Les financiers tricolores, bonapartistes, orléanistes honteux forment le pacte de famine pour amener une restauration monarchique. ${ }^{47}$

Entre le blanc monarchiste et le bleu républicain, entre le rouge internationaliste et le tricolore patriotique, entre le jaune des traitres et le noir de la misère ou de l'obscurantisme, que d'interprétations possibles! « Tout drapeau se prête à discours et surlectures et porte en lui sa future mythologie. » (Pastoureau, 2004, p. 95.)

\section{Références}

BARBIER P. et VERNILLATF., 1961, Histoire de France par les chansons, Paris, NRFGallimard.

BIÉTRY P., 1906, Les Jaunes de France et la question ouvrière, Paris, Paul Paclot. BROSSAT A. et Potel J.-Y., 1975, Antimilitarisme et révolution, Paris, UGE 10/18. CARASSUSÉ., 1982, Les grèves imaginaires, Paris, CNRS.

CAZALS R., 1978, Avec les ouvriers de Mazamet, Paris, Maspero.

CROZETR., 1991, Les instituteurs de Seine-et-Oise vers 1900, Saint-Ouen-L'Aumône, Musée départemental de l'éducation.

DAUDET A., 1898, Soutien de famille, mœurs contemporaines, Paris, Fasquelle.

DUBOIS J., 1962, Le vocabulaire politique et social de 1869 à 1872, Paris, Larousse.

DUCKETT W. éd., 1865 [ $2^{\mathrm{e}}$ éd.], Dictionnaire de la conversation et de la lecture, Paris.

GEFFROY A., 1989, "Curé rouge, faux désignant, vrai concept », Mots. Les langages du politique, $\mathrm{n}^{\circ} 19$, juin, p. 102-106.

MERMEIX (Terail G., dit-), 1907, Le syndicalisme contre le socialisme, Paris, Ollendorff. PARIZE R., 1977, « Les militants ouvriers au Creusot pendant les grèves de 1899-1900 », Le Mouvement social, $\mathrm{n}^{\circ}$ 99, p. 97-108.

45. "Les membres de ce syndicat employaient parfois le terme de jaunes pour désigner leur organisation. [...] On disait aussi syndicats indépendants ou blancs ou tricolores. Leurs drapeaux étaient en effet tricolores. » (Cazals, 1978, p. 59.)

46. "C'est en sortant du régiment que des hommes sont capables de faire des traitres à la classe ouvrière en devenant des policiers ou des jaunes. [...] Voilà où aboutissent finalement les grandes tirades sur la Patrie, les phrases ronflantes sur le Drapeau. » (Nouveau manuel du soldat, CGT, 1902, cité dans Brossat et Potel, 1975, p. 151)

47. La Bataille, 8 décembre 1883. 
PASTOUREAU M., 2000, Bleu. Histoire d'une couleur, Paris, Le Seuil.

2004 [1992], Dictionnaire des couleurs de notre temps, nouvelle édition revue, Paris, Bonneton.

PERROT M., 1974, Les ouvriers en grève (France, 1871-1890), Paris-La Haye, Mouton.

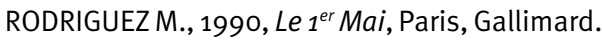

STERNHELLZ., 1978, La droite révolutionnaire (1885-1914), Paris, Le Seuil.

TAGUIEFF P.-A., 1998, La couleur et le sang. Doctrines racistes à la française, Paris, Mille et une nuits.

TOURNIER M., 2002, Propos d'étymologie sociale, 3 vol., Lyon, ENS Éditions.

TILLIER B., 1997, La Républicature, Paris, CNRS.

WARTELLE J.-C., 1993, Les tribulations de Gadlu, grand architecte de son état. Le problème de Dieu dans la franc-maçonnerie, Le Mans, Borrégo.

WILLARD C., 1967, Socialisme et communisme français, Paris, Armand Colin. 